\title{
Calcium blood level modulates endogenous nitric oxide action: effects of parathyroidectomy in patients with hyperparathyroidism
}

\author{
V Martina, G A Bruno ${ }^{1}$, V Brancaleoni, E Zumpano, M Tagliabue, \\ R Fornengo, G Gasparri ${ }^{2}$ and G P Pescarmona ${ }^{1}$
}

Divisione di Endocrinologia, Dipartimento di Medicina Interna, Università degli Studi di Torino, Ospedale Molinette, Corso A M Dogliotti 14 , 10126 Torino, Italy, ${ }^{1}$ Dipartimento di Genetica, Biologia e Chimica Medica, Università degli Studi di Torino, Via Santena 5 bis, 10126 Torino, Italy, and ${ }^{2}$ III Divisione di Chirurgia Generale, Dipartimento di Fisiopatologia Clinica, Università degli Studi di Torino, Ospedale Molinette-Via Genova 3-10126 Torino, Italy

(Requests for offprints should be addressed to V Martina, Divisione di Endocrinologia, Dipartimento di Medicina Interna, Università degli Studi di Torino, Ospedale Molinette, Corso A M Dogliotti 14, 10126 Torino, Italy)

\begin{abstract}
Platelet cyclic guanosine monophosphate (cGMP) is produced by soluble guanylate cyclase (sGC), the activity of which is modulated by the activity of nitric oxide (NO) constitutive synthase (cNOS) which, in turn, is activated by a calcium/calmodulin complex. In primary hyperparathyroidism (H-PTH) an increase in platelet free calcium levels is present. In this study we evaluate the platelet cGMP levels, as an expression of NO production, in the presence of 3-isobutyl-1-methylxanthine (IBMX) alone (IBMXcGMP) and after stimulation by ionomycine (IONO; IONOcGMP) and sodium nitroprusside (SNP; SNPcGMP), in eight subjects affected by H-PTH before and after removal of adenoma. Platelet cGMP levels were also measured in seven normal subjects. IBMXcGMP and IONOcGMP were elevated in H-PTH patients compared with normal subjects $\left(1.9 \pm 0.3\right.$ vs $0.8 \pm 0.2 \mathrm{fmol} / 10^{6}$ platelets and $2 \cdot 7 \pm 0.4$ vs $1 \cdot 4 \pm 0 \cdot 3 ; P<0 \cdot 02$ and $P<0.05$ respectively) but SNPcGMP was unaffected $(3 \cdot 9 \pm 0 \cdot 6$ vs $2 \cdot 5 \pm 0 \cdot 5)$. After parathyroidectomy, blood levels of intact parathyroid hormone (i-PTH), total calcium
\end{abstract}

(t-Ca), IBMXcGMP and IONOcGMP all decreased $(177 \cdot 5 \pm 23.9$ vs $45 \cdot 0 \pm 8.8 \mathrm{pg} / \mathrm{ml}, P<0 \cdot 005 ; 6.5 \pm 0.5$ vs $4.6 \pm 0.1 \mathrm{mEq} / 1, \quad P<0.005 ; 1.9 \pm 0.3$ vs $0.8 \pm 0.2$, $P<0.005 ; 2.7 \pm 0.4$ vs $1.8 \pm 0.3, P<0.05$ respectively), while SNPcGMP was not modified $(3 \cdot 9 \pm 0 \cdot 6$ vs $4 \cdot 3 \pm 0 \cdot 9) . \quad \mathrm{t}-\mathrm{Ca}$ and i-PTH were directly correlated with IBMXcGMP $\left(P<0.02, \quad r_{s}=0.613 ; \quad P<0.02, \quad r_{s}=\right.$ 0.576 respectively) and i-PTH was also correlated with t-Ca $\left(P<0 \cdot 001, r_{s}=0 \cdot 840\right)$. In conclusion: (1) levels of IBMXcGMP and IONOcGMP are high in subjects with H-PTH; (2) after surgery both IBMXcGMP and IONOcGMP decrease to normal values. As IBMXcGMP expresses basal cGMP and IONOcGMP expresses the cGMP after cNOS stimulation, it can be speculated that the increase in NO production could be a mechanism to downregulate the vasoconstriction which may be caused by the high calcium levels in smooth muscle cells. After surgery, together with the normalization of calcium levels, NO production also returned to normal values.

Journal of Endocrinology (1998) 156, 231-235

\section{Introduction}

Cyclic guanosine monophosphate (cGMP) in the platelet is produced by soluble guanylate cyclase (sGC), the activity of which is modulated by nitric oxide (NO) levels. Nitric oxide is synthesized by an enzyme, constitutive NO synthase (cNOS), which converts the amino acid L-arginine to NO and citrulline (Palmer et al. 1988, Palmer \& Moncada 1989). cNOS is calcium/calmodulin-dependent (Mayer et al. 1989) and it releases NO for short periods in response to receptor or physical stimulation (Mulsch et al. 1989, Moncada et al. 1991). NO acts on vascular smooth muscle (Rapoport \& Murad 1983) and platelets (Mellion et al. 1981) through the stimulation of sGC that converts guanosine triphosphate (GTP) to cGMP. The cyclic nucleotide causes smooth muscle relaxation, chiefly via a decrease in intracytosolic calcium (Fig. 1) which, in turn, leads to a decrease in the contractility of the calciumdependent apparatus (Waldman \& Murad 1987, Lincoln et al. 1990). NO synthesis occurs in response to a variety of substances such as the calcium ionophore, ionomycine (IONO) (Furchgott 1984) that forms calcium-permeant channels in the plasma membrane (Knowles et al. 1989), and some nitrovasodilators, such as sodium nitroprusside (SNP) which spontaneously releases $\mathrm{NO}$ which activates sGC (Feelisch \& Noack 1991). In primary hyperparathyroidism (H-PTH) a variable grade of hypercalcemia and an increase in platelet free calcium levels are present. The aim of this study was to assess the activity and regulation of enzymes involved in $\mathrm{NO}$ and cGMP production in 


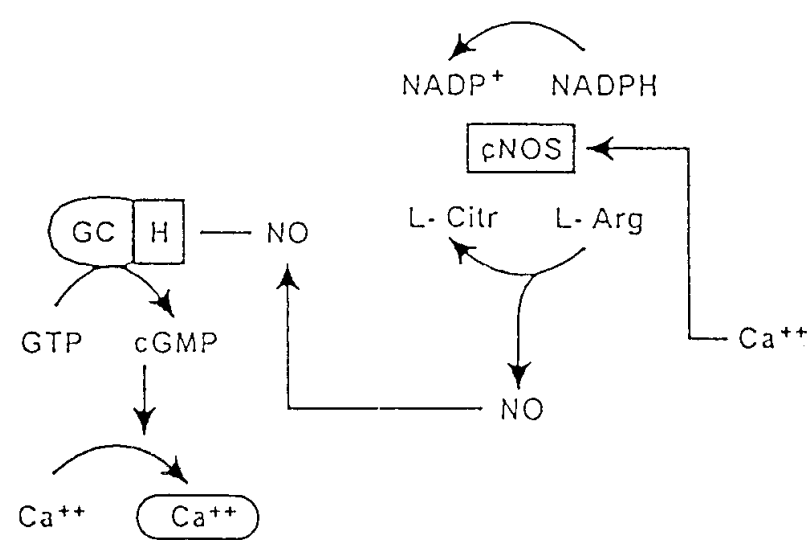

Figure 1 Scheme demonstrating cyclic guanosine monophosphate production. $\mathrm{Ca}^{++}$, free calcium; $\mathrm{H}$, heme; L-Citr, L-citrulline; L-Arg, L-arginine; $\operatorname{NADP}(\mathrm{H})$, nicotinamide adenine dinucleotide phosphate. Other abbreviations as in the text.

platelets of subjects affected by H-PTH before and after removal of adenoma. We evaluated platelet cGMP in three different conditions: (1) with 3-isobutyl-1-methylxanthine (IBMX), an inhibitor of phosphodiesterase, to make cGMP measurable, (2) with IBMX and IONO that induces a maximum calcium influx from the outside and hence a maximal stimulation of the $\mathrm{Ca}^{++}$-dependent cNOS activity, and (3) with IBMX and SNP, an NO donor, that maximally stimulates sGC and can therefore be used as an indirect measurement of active sGC (Fig. 2).

\section{Subjects and Methods}

Eight normotensive (WHO criteria) patients (four females and four males, aged $61 \cdot 4 \pm 2 \cdot 5$ years, body mass index (BMI) $24.8 \pm 0.9 \mathrm{~kg} / \mathrm{m}^{2}$, mean \pm s.E.M.) with $\mathrm{H}-\mathrm{PTH}$ were studied after giving informed consent. The clinical suspicion of H-PTH was confirmed by the laboratory data showing elevated blood levels of intact parathyroid hormone (i-PTH), alkaline phosphatase (ALP) and total calcium ( $\mathrm{t}-\mathrm{Ca})$, while phosphate blood levels $(\mathrm{P})$ tended to be low (see Table 1). The localization of parathyroid adenomas was obtained by scintiscan and ultrasonography studies. Six patients presented with adenoma and two with hyperplasia. Surgical correction of $\mathrm{H}-\mathrm{PTH}$ was obtained in all cases and the typical histological features of parathyroid adenoma were demonstrated. In all patients blood samples were withdrawn at $0800 \mathrm{~h}$ for determination of platelet cGMP, i-PTH, ALP, t-Ca and $\mathrm{P}$ and sampling was repeated 45 days after removing the parathyroid adenoma. cGMP was also evaluated in seven normal subjects (four females and three males, aged $58 \cdot 9 \pm 1 \cdot 2$ years, BMI $23 \cdot 9 \pm 0 \cdot 5)$. Plasma i-PTH $(1-84)$ was measured by the Incstar SP (Incstar Corporation, Stillwater, Minnesota, USA) immunoradiometric assay (IRMA) which utilizes

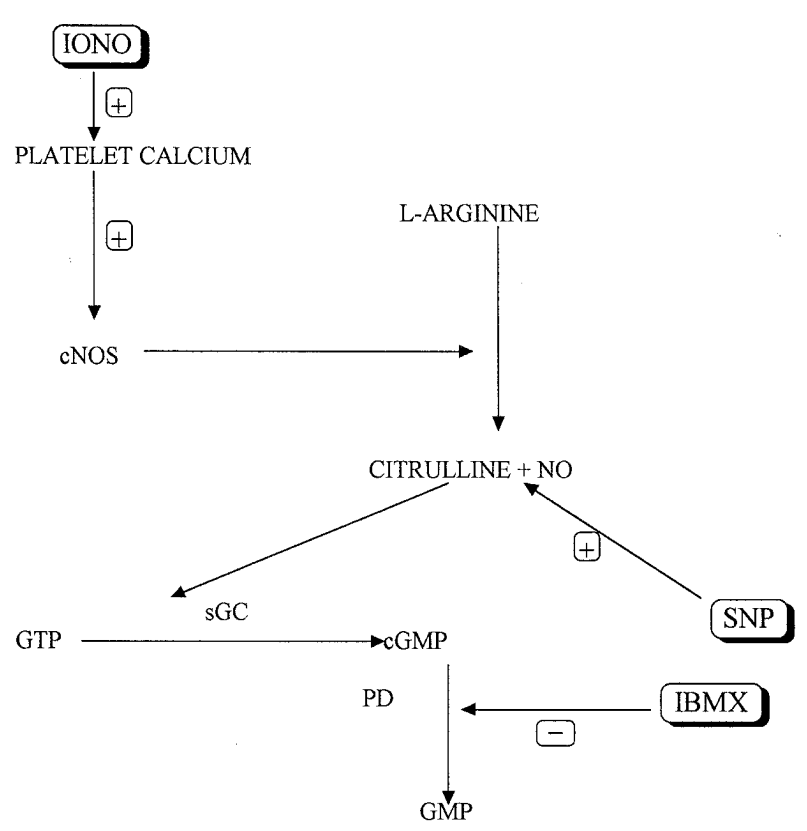

Figure 2 Simplified scheme of drug influence on cGMP production. PD, phosphodiesterase. Other abbreviations as in the text.

two different polyclonal antibodies purified by affinity chromatography. These purified antibodies are specific for two different regions of the PTH molecule. The interassay variation was $4.9 \%$.

Platelet cGMP has been evaluated in three different conditions: (1) with IBMX (Sigma Chemical Co., St Louis, MO, USA), (2) with IBMX and IONO (Calbiochem, San Diego, CA, USA), and (3) with IBMX and SNP (Sigma Chemical Co.). For these measurements the blood samples were collected in one-tenth volume of $3 \cdot 8 \%$ trisodium citrate in plastic tubes. Platelet-rich plasma (PRP) (pH 7.6) was prepared by centrifugation at room temperature for $15 \mathrm{~min}$ at $200 \mathrm{~g}$. The platelet PRP quantification was performed by an impedance method with Coulter S.T.S. Microdiff 18. For the determination of platelet cGMP content, PRP was preincubated at $37^{\circ} \mathrm{C}$ ( $30 \mathrm{~min}$ ) in the presence of $0.5 \mathrm{mM}$ IBMX, then three aliquots were incubated for $15 \mathrm{~min}$ at $37^{\circ} \mathrm{C}$, one as control (IBMX alone) and the other two after the addition of $1 \mathrm{mM}$ SNP or $2 \mu \mathrm{M}$ IONO. After incubation, ice-cold trisodium citrate was added to PRP, platelets were quickly pelleted by centrifugation and the plasma was aspirated; then $0.3 \mathrm{ml} 96 \%$ ethanol was added to the tubes. The ethanol was evaporated and the samples were frozen and kept at $-80^{\circ} \mathrm{C}$ until analysis. The amount of platelet cGMP was measured by a cGMP $\left[{ }^{3} \mathrm{H}\right]$ assay kit provided by Amersham International plc (Amersham, Bucks, UK) and the results were expressed as fmoles cGMP $/ 10^{6}$ platelets. The sensitivity of the assay was $0.5 \mathrm{pmol} / 100 \mu \mathrm{l}$ and the intra- and interassay coefficients of variation were 
Table 1 Blood levels (means \pm S.E.M.) of i-PTH, ALP, total calcium and phosphate in patients with hyperparathyroidism before and after surgery. Normal values are shown in parentheses

\begin{tabular}{|c|c|c|c|c|}
\hline & $\begin{array}{l}\text { i-PTH } \\
(10-65 \mathrm{pg} / \mathrm{ml})\end{array}$ & $\begin{array}{l}\text { ALP } \\
(40-145 \text { U/I) }\end{array}$ & $\begin{array}{l}\text { Total calcium } \\
(4 \cdot 4 \pm 5 \cdot 2 \mathrm{mEq} / \mathrm{l})\end{array}$ & $\begin{array}{l}\text { Phosphate } \\
(0 \cdot 6-2 \cdot 1 \mathrm{mmol} / \mathrm{l})\end{array}$ \\
\hline Before surgery & $177 \cdot 5 \pm 23 \cdot 9$ & $173 \cdot 4 \pm 28 \cdot 8$ & $6 \cdot 5 \pm 0.5$ & $0 \cdot 8 \pm 0 \cdot 1$ \\
\hline After surgery & $45 \cdot 0 \pm 8 \cdot 8$ & $116 \cdot 0 \pm 14 \cdot 5$ & $4 \cdot 6 \pm 0 \cdot 1$ & $1 \cdot 3 \pm 0 \cdot 2$ \\
\hline$P$ value & $<0.005$ & $<0.005$ & $<0.005$ & $<0.005$ \\
\hline
\end{tabular}

between $6 \cdot 3$ and $13 \cdot 1 \%$ and between $6 \cdot 2$ and $19 \cdot 4 \%$ respectively.

All data in text, tables and figures are presented as means \pm s.E.M. Statistical analyses were carried out using non parametric two-way analysis of variance and Spearman's rank correlation coefficient when appropriate.

\section{Results}

Forty-five days after parathyroidectomy levels of i-PTH, ALP and t-Ca had decreased compared with basal values $(177 \cdot 5 \pm 23 \cdot 9$ vs $45 \cdot 0 \pm 8 \cdot 8 \mathrm{pg} / \mathrm{ml} \quad(P<0 \cdot 005)$, $173 \cdot 4 \pm 28 \cdot 8$ vs $116 \cdot 0 \pm 14 \cdot 5 \mathrm{UI} / 1(P<0 \cdot 005), 6 \cdot 5 \pm 0 \cdot 5$ vs $4.6 \pm 0.1 \mathrm{mEq} / / 1 \quad(P<0.005)$ respectively) while $\mathrm{P}$ levels had increased $(0 \cdot 8 \pm 0 \cdot 1$ vs $1 \cdot 3 \pm 0.2 \mathrm{mmol} / 1$ $(P<0 \cdot 005))($ Table 1$)$.

The concentrations of platelet IBMXcGMP and IONOcGMP were elevated in patients affected by H-PTH compared with normal subjects $(1.9 \pm 0.3$ vs $0 \cdot 8 \pm 0.2 \mathrm{fmol} / 10^{6}$ platelets $(P<0 \cdot 02)$ and $2 \cdot 7 \pm 0.4$ vs $1 \cdot 4 \pm 0 \cdot 3(P<0 \cdot 05)$ respectively), but SNPcGMP was not modified (3.9 \pm 0.6 vs $2 \cdot 5 \pm 0 \cdot 5)$ (Fig. 3 ).

The concentrations of platelet IBMXcGMP and IONOcGMP decreased after surgical correction of parathyroid adenomas $\left(1 \cdot 9 \pm 0 \cdot 3 \mathrm{fmol} / 10^{6}\right.$ platelets vs $0 \cdot 8 \pm 0 \cdot 2(P<0 \cdot 005)$ and $2 \cdot 7 \pm 0 \cdot 4$ vs $1 \cdot 8 \pm 0 \cdot 3(P<0 \cdot 05)$ respectively) while the SNPcGMP concentration did not change after adenoma removal $(3.9 \pm 0.6$ vs $4.3 \pm 0.9)$ (Fig. 3).

cGMP increase after SNP addition (SNPcGMP IBMXcGMP) was higher compared with the increase after IONO addition (IONOcGMP - IBMXcGMP) in normal $(1 \cdot 7 \pm 0.4$ vs $0.6 \pm 0 \cdot 1 \quad(P<0 \cdot 01))$ and in H-PTH patients before and after surgery $(3.5 \pm 1 \cdot 0$ vs $0 \cdot 9 \pm 0 \cdot 3(P<0 \cdot 05)$ and $1.9 \pm 0.7$ vs $0.8 \pm 0 \cdot 2(P<0 \cdot 01)$ respectively).

Total Ca was correlated with basal cGMP (IBMXcGMP; $\left.P<0.02, r_{s}=0.613\right)$ in the whole population. i-PTH was also correlated with IBMXcGMP $(P<0 \cdot 02$, $\left.\mathrm{r}_{\mathrm{s}}=0 \cdot 576\right)$ and with $\mathrm{t}-\mathrm{Ca}\left(P<0 \cdot 001, \mathrm{r}_{\mathrm{s}}=0 \cdot 840\right)$.

\section{Discussion}

This study demonstrates that platelet cGMP, both basal (IBMXcGMP) and after stimulation with ionomycine
(IONOcGMP), is increased in subjects affected by $\mathrm{H}-\mathrm{PTH}$ compared with normal subjects and it decreases to normal values after surgery of adenoma. cGMP in the platelet is produced by sGC, the activity of which is modulated by the NO levels; hence, the increase in IBMXcGMP could be due to an increase in sGC activity and/or to an increase in cNOS activity (i.e. by an increase in NO production). SNPcGMP levels, before and after surgery, are superimposable suggesting that the sGC activity is not affected by $\mathrm{H}-\mathrm{PTH}$, while the elevated IONOcGMP levels suggest that H-PTH is able to increase cNOS activity. Furthermore, the cGMP increase after SNP addition, both in normal subjects and in patients with $\mathrm{H}-\mathrm{PTH}$, is significantly higher than the cGMP increase after IONO addition, excluding a limiting role of sGC in the regulation of cGMP level in the platelets.

It is well known that a diet rich in calcium content is able to reduce the blood pressure in young people with mild hypertension (Grobbee \& Hofman 1986). This finding was confirmed in spontaneously hypertensive rats, with a significant positive correlation between systolic blood pressure and PTH (Rao et al. 1994). On the other hand some evidence indicates that in $\mathrm{H}-\mathrm{PTH}$ the intraplatelet calcium levels are increased. Calcium may activate calcium/calmodulin-dependent cNOS, increasing NO production. Furthermore, PTH has an acute hypotensive effect (Mok et al. 1989) in spontaneously hypertensive Wistar-Kyoto rats, which persists in the presence of an inhibitor of $\mathrm{NO}$ synthesis, indicating that the hypotensive response to $\mathrm{PTH}$ is $\mathrm{NO}$-independent (Schleiffer et al. 1995). Certainly H-PTH affects platelet calcium by increasing extracellular calcium, the increased gradient between extracellular and intracellular calcium eliciting a greater calcium entry (Kawashima 1990). Actually, it has been demonstrated that normotensive patients with H-PTH have elevated concentrations of intraplatelet calcium that correlate well with blood PTH concentrations and return to normal after correction of the H-PTH condition (Fardella \& Rodriguez-Portales 1995). Assuming a high basal intraplatelet calcium in our H-PTH patients, we can explain the higher levels of IBMXcGMP in resting platelets and the correlation of these values with blood calcium and PTH. Taken together these data suggest that the hypotensive action of calcium could be mediated by an enhancement of NO production. On this basis we suggest that the increased levels of IBMXcGMP 

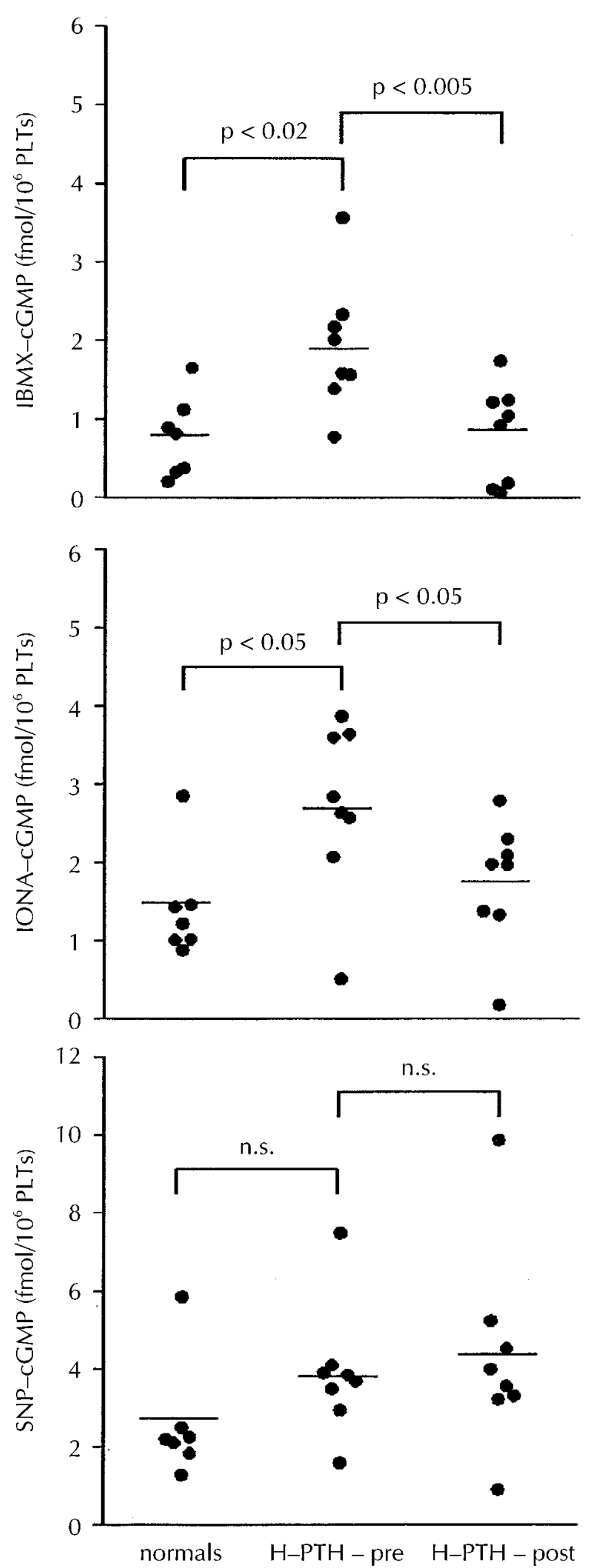

Figure 3 Platelet cGMP levels in normal subjects and in patients affected by hyperparathyroidism before (H-PTH-pre) and after (H-PTH-post) surgery. PLTs, platelets; n.s., not significant. and IONOcGMP in H-PTH normotensive patients counteract calcium action on the contractility of the smooth muscle preventing the development of arterial hypertension. In agreement with this hypothesis it has been demonstrated that in essential hypertension the intracellular calcium is elevated (Fardella \& RodriguezPortales 1995) but that the endothelium-dependent NO-induced cGMP production is decreased (Otsuka et al. 1985, Shirasaki et al. 1988). Finally, since arterial hypertension has been reported in up to $30-40 \%$ of patients with primary hyperparathyroidism (Lafferty 1986), we may suppose that in these patients, as in patients with essential hypertension, NO production is not sufficient to counteract the contraction of the smooth muscle cells due to the increase in $\mathrm{Ca}^{++}$cell content. On the other hand the presence of a parathyroid hypertensive factor in $\mathrm{H}-\mathrm{PTH}$ patients with high blood pressure cannot be ruled out.

In conclusion, our data has demonstrated for the first time that: (1) cGMP is higher in normotensive subjects with H-PTH, (2) cGMP, both basal and following stimulation by ionomycine, decreases after surgery. As our $\mathrm{H}-\mathrm{PTH}$ patients were normotensive and assuming that platelet cGMP levels are an expression of cNOS activity it can be speculated that the increase in NO production (by platelets and possibly also by endothelial cells) is a mechanism able to counteract the vasoconstriction caused by the high calcium levels in the smooth muscle cells. After surgery, together with normalization of PTH and $\mathrm{t}$-Ca levels, cGMP and hence NO production returned to normal values suggesting a role of calcium and PTH in the regulation of cNOS activity. Further studies are necessary to understand the mechanism by which patients with H-PTH develop arterial hypertension.

\section{Acknowledgement}

This work has been partially supported by a grant from the MURST.

\section{References}

Fardella C \& Rodriguez-Portales JA 1995 Intracellular calcium and blood pressure: comparison between primary hyperparathyroidism and essential hypertension. Journal of Endocrinological Investigation 18 827-832.

Feelisch M \& Noack E 1991 The in vitro metabolism of nitrovasodilators and their conversion into vasoactive species. In Heart Failure - Mechanism and Management, pp 241-255. Eds BS Lewis \& A Kimchi. Berlin: Springer Verlag.

Furchgott RF 1984 The role of endothelium in the responses of vascular smooth muscle to drugs. Annual Review of Pharmacology and Toxicology 24 175-197.

Grobbee DE \& Hofman A 1986 Effect of calcium supplementation on diastolic blood pressure in young people with mild hypertension. Lancet ii 703-707.

Kawashima H 1990 Parathyroid hormone causes a transient rise in intracellular ionized calcium in vascular smooth muscle cells. Biochemical and Biophysical Research Communications 166 709-711. 
Knowles RG, Palacios M, Palmer RMJ \& Moncada S 1989 Formation of nitric oxide from 1 -arginine in the central nervous system: a transduction mechanism for stimulation of the soluble guanylate cyclase. Proceedings of the National Academy of Sciences of the USA $\mathbf{8 6}$ 5159-5162.

Lafferty F 1986 Primary hyperparathyroidism. Changing clinical spectrum, prevalence of hypertension and discriminant analysis of laboratory tests. Archives of Internal Medicine 141 1761-1765.

Lincoln TM, Cornwell TL \& Taylor AE 1990 cGMP-dependent protein kinase mediates the reduction of $\mathrm{Ca}^{2+}$ by cAMP in vascular smooth muscle cells. American Journal of Physiology 258 C399-C407.

Mayer B, Schmidt K, Humbert R \& Bohme E 1989 Biosynthesis of endothelium-derived relaxing factor: a cytosolic enzyme in porcine aortic endothelial cells $\mathrm{Ca}^{++}$-dependently converts L-arginine into an activator of soluble guanylyl cyclase. Biochemical and Biophysical Research Communications 164 678-685.

Mellion BT, Ignarro BJ, Ohlstein EH, Pontecorvo EG, Hyman AL \& Kadowitz PJ 1981 Evidence for inhibitory role of guanosine $3^{\prime}, 5^{\prime}$ monophosphate in ADP induced human platelet aggregation in the presence of nitric oxide and related vasodilators. Blood $\mathbf{5 7}$ 946-955.

Mok LLS, Nickols GA, Thompson JA \& Cooper CW 1989 Parathyroid hormone as a smooth muscle relaxant. Endocrine Reviews $10420-436$.

Moncada S, Palmer RMJ \& Higgs EA 1991 Nitric oxide: physiology, pathophysiology and pharmacology. Pharmacological Review $\mathbf{4 3}$ 109-141.

Mulsch A, Bassenge E \& Busse R 1989 Nitric oxide synthesis in endothelial cytosol: evidence for a calcium-dependent and a calcium-independent mechanism. Naunyn-Schmiedeberg's Archives of Pharmacology $340767-770$.
Otsuka Y, Di Piero A, Hirt E, Brennaman B \& Lockette W 1985 Vascular relaxation and cGMP in hypertension. American Journal of Physiology 254 163-169.

Palmer RMJ \& Moncada S 1989 A novel citrulline-forming enzyme implicated in the formation of nitric oxide by vascular endothelial cells. Biochemical and Biophysical Research Communications $\mathbf{1 5 8}$ 348-352.

Palmer RMJ, Rees DD \& Ashton DS 1988 L-Arginine is the physiological precursor for the formation of nitric oxide in endothelium-dependent relaxation. Biochemical and Biophysical Research Communications 153 1251-1256.

Rao RM, Yan Y \& Wu Y 1994 Dietary calcium reduces blood pressure, parathyroid hormone and platelet cytosolic calcium responses in spontaneously hypertensive rats. American Journal of Hypertension 7 1052-1057.

Rapoport RM \& Murad F 1983 Agonist induced endotheliumdependent relaxation in rat thoracic aorta may be mediated through cyclic GMP. Circulation Research 52 352-357.

Schleiffer R, Pernot F \& Gairard A 1995 Endogenous nitric oxide is not involved in acute parathyroid hormone-induced hypotension in rat: effect of hypertension and hypoparathyroid status. Hormone and Metabolic Research 27 415-418.

Shirasaki Y, Kolm P, Nickols GA \& Lee TJF 1988 Endothelial regulation of cyclic GMP and vascular responses in hypertension. Journal of Pharmacology and Experimental Therapeutics 245 53-58.

Waldman SA \& Murad F 1987 Cyclic GMP synthesis and function. Pharmacological Reviews 39 163-196.

Received 19 May 1997

Revised manuscript received 30 July 1997

Accepted 26 August 1997 